\title{
A Theoretical Study of the Performance Improvement in GSM Networks Due to Slow Frequency Hopping
}

\author{
A Sathyendran ${ }^{*}$ and $\mathbf{P} \mathbf{J}$ Smith \\ * - BellSouth New Zealand, \# - Victoria University of Wellington, New Zealand \\ BellSouth New Zealand, Level 6, 21 Pitt Street, Private Bag 92161, Auckland, New Zealand \\ Ph. No. +649357 0326, Fax No. +64 93793451 , \\ Email : arasaratnam.sathyendran@bellsouth.co.nz
}

\begin{abstract}
This paper presents a theoretical method to study the performance improvement in GSM networks due to slow frequency hopping when a limited number of hopping channels are available. By comparing the performance improvement offered by frequency hopping with intra-timeslot handover, new design thresholds and capacity gain figures are derived for GSM networks.

The analysis shows that to gain an advantage from frequency hopping it is necessary to deploy more than two hopping channels. Frequency hopping enables the network designer to reduce the $\mathrm{C} / \mathrm{I}$ protection margin by about $5 \mathrm{~dB}$ when the channel occupancy is low and by about $3 \mathrm{~dB}$ when the channel occupancy is high. This reduction in protection margin results in the improvement in spectral efficiency of $82 \%$ when channel occupancy is low and $54 \%$ when channel occupancy is high. Frequency hopping can be used to improve the network quality where significant improvements can be achieved. The improvement in network quality depends on the channel occupancy.
\end{abstract}

\section{Introduction}

Frequency hopping is one of the many techniques that are being considered to improve the spectral efficiency of GSM cellular networks. To gain the maximum benefit from frequency hopping it is necessary to modify the traditional design parameters to take into account the effect of frequency hopping. This paper presents a theoretical method for determining the new design parameters.

A number of studies have been carried out to quantify performance improvement in GSM networks due to slow frequency hopping $[1,2]$. Generally these studies have assumed that there are a large number of hopping channels and this has enabled the use of various approximations that can be applied with large numbers. However, in practical networks there are only a limited number of channels available and the accuracy of these approximations are questionable. A previous paper [3] studied the performance improvement in GSM networks due to slow frequency hopping when a limited number of hopping channels were available. In this paper simulation techniques were used and the model developed was specific to the frequency reuse factor used. In this paper this model is generalised to made non specific to the frequency reuse factor.

This paper is organised as follows. The system model and the method of analysis are presented in Section II. The results and the parameters used in the calculation of results are outlined in Section III. Conclusions are presented in Section IV. The derivation of probability density function (pdf) of carrier to interference ratio $(\mathrm{C} / \mathrm{I})$ is presented in the Appendix.

\section{System Model and Method of Analysis}

Frequency hopping improves the system performance by providing immunity to fast fading (Rayleigh fading [4]) and cochannel interference. Fast fading in the mobile radio environment is caused by multi-path propagation of the signal by local scatterers. The path length as a multiple of the wavelength is different at different frequencies. Due to this difference the fading experienced by different frequencies varies at a particular location. By continuously changing the carrier frequency, hopping reduces the duration of deep fades. Similarly frequency hopping also reduces the duration of severe interference. The immunity provided by frequency hopping depends on the number of hopping channels and the correlation bandwidth of the channel. In this paper it is assumed that the consecutive hopping channels are uncorrelated. (i.e. consecutive hopping channels are separated by more than the correlation bandwidth).

In cellular environments the signal undergoes fast fading, shadowing and path loss. In this paper the performance of a mobile at the cell boundary is considered. At a particular location the signal variation due to shadow fading is negligible or minimal. Therefore in this analysis the effect of shadow fading is ignored. However, in the determination of cell boundaries a protection margin is added to take into account the log-normal shadowing.

In order to estimate the improvement offered by frequency hopping the $\mathrm{C} / \mathrm{I}$ is estimated as a function of the number of hopping channels. In GSM speech bursts are interleaved over eight bursts, thus the bit error rate of the transmitted information depends on the mean carrier to interference ratio (C/I) of the eight bursts rather than on that of individual bursts [5]. It is assumed that the channel conditions do not change over the period when the eight bursts are received. The average $\mathrm{C} / \mathrm{I}$ (power) is given by
VTC ‘98 


$$
\mathrm{C} / \mathrm{I}=\frac{1}{n} \sum_{i=1}^{n} \frac{\alpha_{i}^{2}}{\mathrm{p} \sum_{k=1}^{L} \alpha_{i k}^{2}}=\frac{\gamma}{n \mathrm{p}},
$$

where $\alpha_{i}$ is the desired signal voltage of the ith hop, $\alpha_{i k}$ is the interference signal from the $j$ th cochannel cell of the $i$ th hop, $n$ is the number of hopping channels, $\mathrm{p}$ is the probability of channel occupancy and $L$ is the number of cochannel interferers. In (1) only one $\mathrm{C} / \mathrm{I}$ sample is taken over the eight burst period. Due to the assumption that the channel condition does not change over the eight burst period only one sample is needed to represent the average $\mathrm{C} / \mathrm{I}$ over the eight burst period. Both $\alpha_{i}$ and $\alpha_{i k}$ are Rayleigh distributed random variables [4]. The Rayleigh distribution is given by $[4,6]$

$$
\operatorname{pdf}(\alpha)=\frac{2 \alpha}{\Gamma} \exp \left[-\frac{\alpha^{2}}{\Gamma}\right],
$$

where $\alpha$ is the received signal voltage and $\Gamma$ is the mean signal power. The mean signal strength can be calculated from

$$
\Gamma=\mathrm{P}_{\mathrm{T}}-\mathrm{L}_{\mathrm{P}}+\mathrm{G},
$$

where $\mathrm{P}_{\mathrm{T}}$ is the transmit power, $\mathrm{L}_{\mathrm{P}}$ is the mean path loss and $G$ is the antenna gain of the transmitter. The Hata model has been used to model the mean path loss [7] i.e.

$$
\begin{aligned}
\mathrm{L}_{\mathrm{p}} & =69.55+26.16 \log \mathrm{f}_{\mathrm{c}}-13.82 \log \mathrm{h}_{\mathrm{t}} \\
& +\left(44.9-6.55 \log \mathrm{h}_{\mathrm{t}}\right) \log d,
\end{aligned}
$$

where $f_{c}$ is the carrier frequency in $M H z, h_{t}$ is the base antenna height in meters, $d$ is the distance between the base station and the mobile in kilometers. The effect of mobile antenna height is ignored. The above expression applies only to urban environments. In this paper only the urban environment is considered. The method presented in this paper is not specific to any environment or frequency reuse factor. The urban environment is used to illustrate the method of analysis.

The probability that the $\mathrm{C} / \mathrm{I}$ is less than the desired threshold $X$, is given by

$$
\operatorname{Prob}(\mathrm{C} / \mathrm{I}<X)=\int_{0}^{n p X} \operatorname{pdf}_{\gamma}(\gamma) d \gamma .
$$

In order to estimate the probability that the $\mathrm{C} / \mathrm{I}$ is less than the desired values it is necessary to derive the pdf of $\gamma$. The derivation of the pdf of $\gamma$ is given in the Appendix.

The effective frequency hopping gain is calculated by comparing it to the performance of intra-timeslot handover. Intra-time slot handover is currently used in GSM networks which uses idle timeslot interference measurements to select the channel with the least interference whenever excess 0-7803-4320-4/98/\$5.00 $\odot 1998$ IEEE interference is experienced by the mobile. The $\mathrm{C} / \mathrm{I}$ is evaluated using the following expression

$$
\mathrm{C} / \mathrm{I}=\sum_{i=1}^{m} \sum_{k=0}^{L}{ }^{L} \mathrm{C}_{k} \mathrm{p}^{k}(1-\mathrm{p})^{L-k} \alpha_{i} / \alpha_{i k},
$$

where $\alpha_{i}$ is the desired signal voltage of the $i$ th measurement received on the SACCH (slow associated control channel) burst, $\alpha_{i k}$ is the signal strength of the $j$ th interferer of the $i$ th SACCH burst, $\mathrm{p}$ is the probability of channel occupancy, $L$ is the number of cochannel interferers and $m$ is the number of $\mathrm{SACCH}$ bursts over which average $\mathrm{C} / \mathrm{I}$ is calculated. Intratimeslot handover is assumed to only occur on the serving base station. In an actual system, intra-timeslot handover will occur on both the serving and interfering cells. As a result collisions will occur and additional intra-timeslot handovers will be attempted. Therefore the performance of the intra-timeslot handover presented in this paper would be the maximum that could be obtained.

\section{Results}

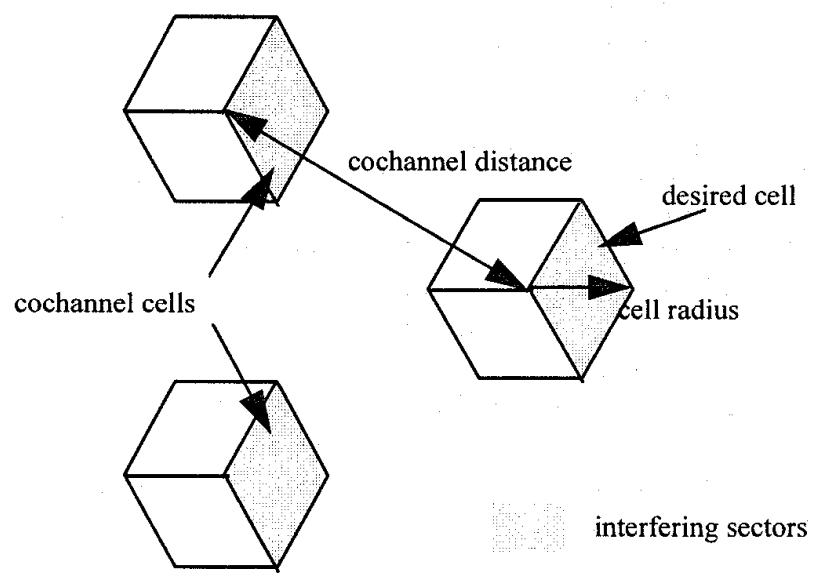

Figure 1 : Position of desired and cochannel sectors.

Figure 1 shows the position of the desired and cochannel sectors. The shaded sectors represent the interfering sectors. In order to calculate the probability that $\mathrm{C} / \mathrm{I}<X$ for different mean $\mathrm{C} / \mathrm{I}$ at the boundary, the cochannel distance was adjusted until the desired mean $\mathrm{C} / \mathrm{I}$ is obtained at the boundary. To estimate the performance of intra-timeslot handover, Rayleigh random variables are generated and the average signal strength is calculated over 6 measurements received in SACCH bursts [5]. This value is compared with a threshold before intratimeslot handover is attempted.

Three channel occupancy figures, $0.45,0.6$ and 0.75 are considered in the calculation of results. Channel occupancy of 0.45 represents channel utilisation when low number of channels are available (e.g. one transceiver with 7 channels). Channel occupancy of 0.6 represents channel utilisation when networks are designed for low blocking (e.g. $2 \%$ ) and when large number of channels are available (e.g. three transceivers with 22 channels). Channel occupancy of 0.75 represents 
channel utilisation when networks are designed for medium blocking (e.g. 5\%).

The parameters used in the calculation of results are presented in Table 1.

Table 1 : Parameters used in the calculation of results.

\begin{tabular}{|c|c|}
\hline Parameter & Value \\
\hline Receiver sensitivity & $-102 \mathrm{dBm}$ \\
\hline Minimum C/I required & $9 \mathrm{~dB}$ \\
\hline $\begin{array}{c}\text { Signal strength at the cell } \\
\text { boundary }\end{array}$ & $-85 \mathrm{dBm}$ \\
\hline Base transmit power & $53 \mathrm{dBm}$ \\
\hline Antenna height & $20 \mathrm{~m}$ \\
\hline Operating frequency & $925 \mathrm{MHz}$ \\
\hline $\begin{array}{c}\text { Intra-timeslot handover } \mathrm{C} / \mathrm{I} \\
\text { trigger threshold }\end{array}$ & $6 \mathrm{~dB}$ \\
\hline
\end{tabular}

Figures 2-4 show the probability that $\mathrm{C} / 1$ is less than $9 \mathrm{~dB}$ at the cell boundary for channel occupancies of $0.45,0.6$ and 0.75 respectively. The probability that $\mathrm{C} / \mathrm{I}$ is less than $9 \mathrm{~dB}$ are calculated for non hopping, two, four, six and eight channel frequency hopping as well as for intra-timeslot handover. The label "Intra" refers to results obtained for intra-timeslot handover. The labels " 1 ", " 2 ", " 4 ", "6" and " 8 " refer to the number of hopping channels used. With a frequency reuse factor of 4 the $\mathrm{C} / \mathrm{I}$ at the boundary is $16.6 \mathrm{~dB}$ (generally a frequency reuse factor of 4 is used in GSM).

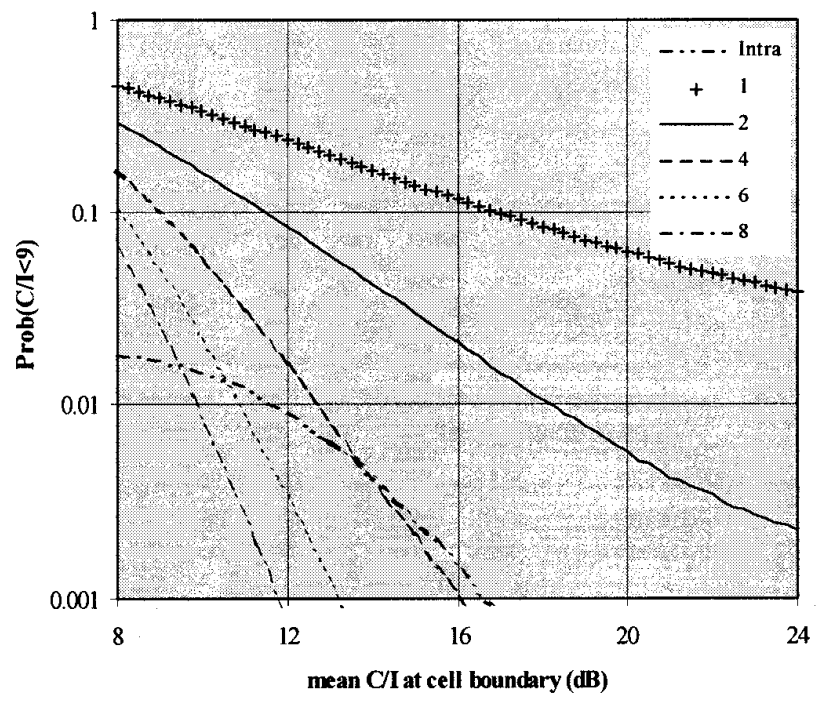

Figure 2 : Probability that the $\mathrm{C} / \mathrm{I}<9 \mathrm{~dB}$ as a function of $\mathrm{C} / \mathrm{I}$ at the cell boundary with a channel occupancy of 0.45 .

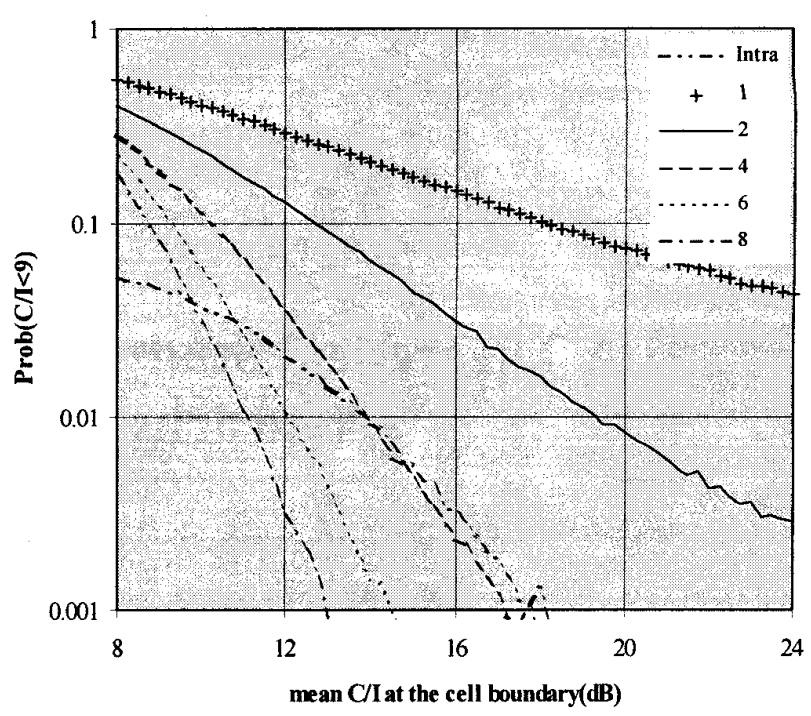

Figure 3 : Probability that the $\mathrm{C} / \mathrm{I}<9 \mathrm{~dB}$ as a function of $\mathrm{C} / \mathrm{I}$ at the cell boundary with a channel occupancy of 0.6 .

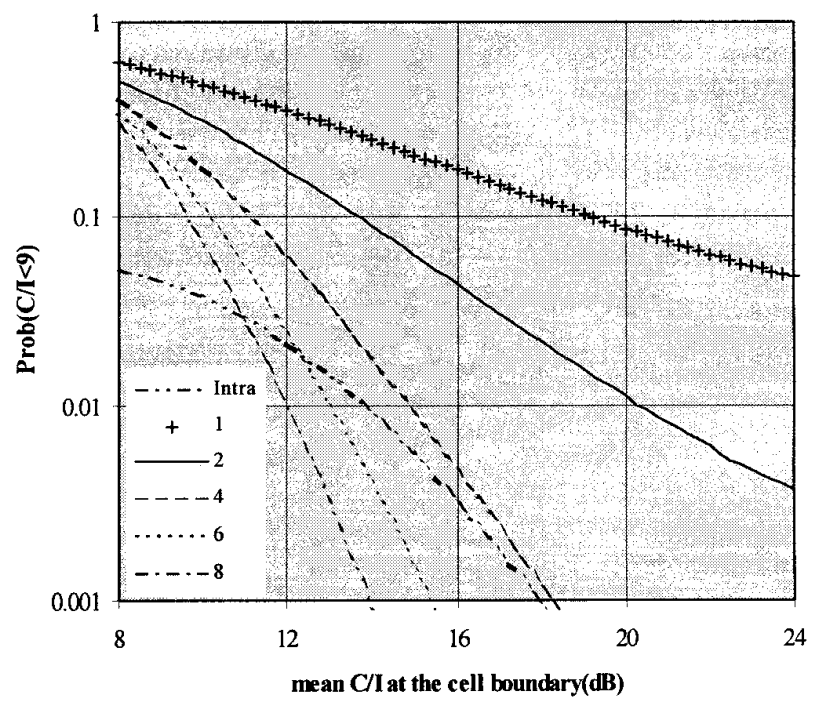

Figure 4 : Probability that the $C / I<9 d B$ as a function of $\mathrm{C} / \mathrm{I}$ at the cell boundary with a channel occupancy of 0.75 .

In the calculation of results a maximum of eight hopping channels are considered. The performance improvement when hopping over more than eight channels will be negligible as consecutive hops are assumed to be uncorrelated. Some interferer diversity can be achieved by hopping over more than eight channels, however this improvement is minimal. However, if the bursts are correlated, improvement may be experienced when more than eight hopping frequencies are used. Theoretically maximum improvement can be achieved when an infinite number of channels is used. 
Frequency hopping allows the use of a lower protection margin in determining the cochannel distances (or tighter frequency reuse than is possible with no hopping). Figures 2-4 can be used to determine the $\mathrm{C} / \mathrm{I}$ margins required at the cell boundary with different number of hopping channels to achieve the same quality offered by the intra-timeslot handover. These $\mathrm{C} / \mathrm{I}$ values required at the cell boundary are tabulated in Table 2 .

Table 2 : C/I required at the boundary as a function of hopping channels and channel occupancy.

\begin{tabular}{|c|c|c|c|}
\hline \multirow{2}{*}{ No. of Hopping Channels } & \multicolumn{3}{|c|}{ Channel Occupancy } \\
\cline { 2 - 4 } & 0.45 & 0.6 & 0.75 \\
\hline 4 & 16 & 16 & 17 \\
\hline 6 & 13 & 13.5 & 14.75 \\
\hline 8 & 11.75 & 12.5 & 13.25 \\
\hline
\end{tabular}

The results presented in Table 2 indicate that the gains from frequency hopping diminish as the channel occupancy increases. The C/I required at the boundary can be reduced by about $5 \mathrm{~dB}$ in low channel occupancy scenarios and by about $3 \mathrm{~dB}$ in high channel occupancy scenarios. The reduction in protection margin at the cell boundary allows the use of tighter frequency reuse factor which results in higher spectral efficiency. The improvement in spectral efficiency (channels/MHz/cell) is given by

$$
\text { improvement }=1-\frac{\mathrm{K}_{\text {no_hopping }}}{\mathrm{K}_{\text {hopping }}}
$$

where $\mathrm{K}_{\text {no hopping }}$ is the frequency reuse factor with no frequency hoping and $K_{\text {hopping }}$ is the frequency reuse factor with frequency hopping. The $\mathrm{C} / \mathrm{I}$ required at the boundary, the reuse factor and the effective frequency hopping capacity improvement are presented in Tables 3-4 for channel occupancies of $0.45,0.6$ and 0.75 respectively.

Table 3 : C/I required at the boundary, the reuse factor and improvement in spectral efficiency as a function of hopping channels for channel occupancy of 0.45 .

\begin{tabular}{|c|c|c|c|}
\hline $\begin{array}{c}\text { No. of Hopping } \\
\text { Channels }\end{array}$ & $\mathrm{C} / \mathrm{I}$ & $\mathrm{K}$ & Improvement (\%) \\
\hline 4 & 16 & 3.7 & 8 \\
\hline 6 & 13 & 2.5 & 60 \\
\hline 8 & 11.75 & 2.2 & 82 \\
\hline
\end{tabular}

$0-7803-4320-4 / 98 / \$ 5.00 \odot 1998$ IEEE
Table $4: \mathrm{C} / \mathrm{I}$ required at the boundary, the reuse factor and improvement in spectral efficiency as a function of hopping channels for channel occupancy of 0.6 .

\begin{tabular}{|c|c|c|c}
\hline $\begin{array}{c}\text { No. of Hopping } \\
\text { Channels }\end{array}$ & $\mathrm{C} / \mathrm{I}$ & $\mathrm{K}$ & Improvement (\%) \\
\hline 4 & 16 & 3.7 & 8 \\
\hline 6 & 13.5 & 2.7 & 48 \\
\hline 8 & 12.5 & 2.3 & 74 \\
\hline
\end{tabular}

Table $5: \mathrm{C} / \mathrm{I}$ required at the boundary, the reuse factor and improvement in spectral efficiency as a function of hopping channels for channel occupancy of 0.75 .

\begin{tabular}{|c|c|c|c|}
\hline $\begin{array}{c}\text { No. of Hopping } \\
\text { Channels }\end{array}$ & $\mathrm{C} / \mathrm{I}$ & $\mathrm{K}$ & Improvement (\%) \\
\hline 4 & 17 & 4.2 & -5 \\
\hline 6 & 14.75 & 3.2 & 25 \\
\hline 8 & 13.25 & 2.6 & 54 \\
\hline
\end{tabular}

Frequency hopping can also be used to improve the network quality. Table 6 outlines the improvement in network quality for different numbers of hopping channels. The network quality is given by the probability that $\mathrm{C} / \mathrm{I}<9 \mathrm{~dB}$ when the $\mathrm{C} / \mathrm{I}$ at the cell boundary is $16.6 \mathrm{~dB}$.

Table 6: Quality improvement as a function of hopping channels for channel occupancy of 0.6 .

\begin{tabular}{|c|c|c|}
\hline $\begin{array}{c}\text { No. of Hopping } \\
\text { Channels }\end{array}$ & $\operatorname{Prob}(\mathrm{C} / \mathrm{I}<9)$ & $10 \log ($ Improvement $)$ \\
\hline Intra-timeslot & 0.0023 & 0 \\
\hline 1 & 0.13 & -17.5 \\
\hline 2 & 0.025 & -10.4 \\
\hline 4 & 0.0016 & 1.6 \\
\hline 6 & 0.00014 & 12.2 \\
\hline 8 & 0.00001 & 23.6 \\
\hline
\end{tabular}

\section{Conclusions}

The performance improvement offered by frequency hopping depends on the number of hopping channels and on channel occupancy. The analysis shows that intra-timeslot handover provides better interference performance compared to two channel hopping. Frequency hopping can be used to reduce the protection margin. The $\mathrm{C} / \mathrm{I}$ required at the boundary can be reduced by about $5 \mathrm{~dB}$ in low channel occupancy scenarios and by about $3 \mathrm{~dB}$ in high channel occupancy scenarios. This reduction in protection margin translates to improvement in spectral efficiency of $82 \%$ when channel 
occupancy is low and $54 \%$ when channel occupancy is high. Significant improvement in network quality can be achieved if frequency hopping is used to improve the network quality. The improvement in quality depends on the number of hopping channels and on channel occupancy.

Further study is required to incorporate the effect of correlation bandwidth on frequency hopping, and the effect of time slot collisions upon intra-timeslot handover performance.

\section{Appendix}

\section{Derivation of PDF of C/I}

In this appendix the pdf of $\gamma$ is derived. The derivation of the pdf of $\gamma$ is done in two steps, first the pdf of $\gamma_{i}$ defined as $\gamma_{i}=\frac{\alpha_{i}^{2}}{\sum_{k=1}^{K} \alpha_{i k}^{2}}$ is derived and from this the pdf of $\gamma$ is derived.

The pdf of $\gamma_{i}$ is obtained using the Mellin convolution [8]. The pdf of $\gamma_{i}$ is given by [9]

$$
\operatorname{pdf}_{\gamma_{i}}\left(\gamma_{i}\right)=\sum_{k=1}^{L} \frac{\frac{\Gamma_{i k}^{L}}{\Gamma_{i}}}{\prod_{\substack{l=1 \\ l \neq k}}^{L}\left(\Gamma_{i k}-\Gamma_{i l}\right)} \frac{1}{\left[\frac{\Gamma_{i k}}{\Gamma_{i}} \gamma_{i}+1\right]^{2}}
$$

The pdf of $\gamma$ is given by

$$
\operatorname{pdf}_{\gamma}(\gamma)=\frac{1}{2 \pi} \int_{-\infty}^{\infty} \Psi(\omega) \exp (-j \omega \gamma) d \omega
$$

where $\Psi(\omega)$ is the characteristic function of $\gamma$ and is given by

$$
\begin{aligned}
\Psi(\omega) & =\int_{-\infty}^{\infty} \operatorname{pdf}(\gamma) \exp (j \omega \gamma) d \gamma=\mathrm{E}[\exp (j \omega \gamma)] \\
& =\prod_{i=1}^{n} \Psi_{i}(\omega)=\left[\Psi_{i}(\omega)\right]^{n},
\end{aligned}
$$

where $\Psi_{i}(\omega)$ is the characteristic function of $\gamma_{i} . \Psi_{i}(\omega)$ can be expressed as a power of $\Psi_{i}(\omega)$ because the desired and interfering signal are independent identically distributed signals. $\Psi_{i}(\omega)$ is given by $[10]$

$$
\Psi_{i}(\omega)=\frac{1}{2 \pi} \int_{-\infty}^{\infty} \operatorname{pdf}_{\gamma_{i}}\left(\gamma_{i}\right) \exp \left(j \omega \gamma_{i}\right) d \gamma_{i}
$$

It can be shown that the expression for $\Psi_{i}(\omega)$ reduces to

$$
\begin{aligned}
\Psi_{i}(\omega) & =\sum_{k=1}^{L} \frac{\frac{\Gamma_{i k}^{L}}{\Gamma_{i}}}{\prod_{\substack{l=1 \\
l \neq k}}^{L}\left(\Gamma_{i k}-\Gamma_{i l}\right)} \times\left[\frac{\Gamma_{i}}{\Gamma_{i k}}+\right. \\
& \left.j \omega\left[\frac{\Gamma_{i}}{\Gamma_{i k}}\right]^{2} \exp \left(-j \frac{\Gamma_{i}}{\Gamma_{i k}} \omega\right) \mathrm{E}_{1}\left[-j \omega \frac{\Gamma_{i}}{\Gamma_{i k}}\right]\right]
\end{aligned}
$$

where $\mathrm{E}_{1}[x]$ is the exponential integral.

\section{References}

[1] Jean-Louis Dornstetter and Didier Verhulst "Cellular Efficiency with Slow Frequency Hopping: Analysis of Digital SFH900 Mobile System," JASAC vol. SAC-5, pp. 835-848, June 1987.

[2] Caisia Carneheim, Sven - Olof Jonsson, Malin Ljungberg, Magnus Madfors and Jonas Naslund "FH GSM Frequency Hopping GSM" Proceedings of IEEE 44th VTC, pp. 1155-1159, Sweden 1994.

[3] G W Tunnicliffe, A Sathyendran and A R Murch, "Performance Improvement in GSM Networks due to Slow Frequency Hopping" Proceedings of IEEE 47th VTC, pp. 1857-1861, Pheonix 1997.

[4] IEEE Vehicular Technology Society Committee on Radio Propagation: "Special issue on mobile radio propagation," IEEE Trans. Vech. Technol., vol. 37, pp. 3-72, February 1988.

[5] Michel Mouly, Marie-Bernadette Pautet "The GSM System for Mobile Communications" CELL \& SYS 1992.

[6] J D Parsons and J G Gardiner, "Mobile Communication Systems," Blackie, Chapter 3, 1989

[7] M Hata, Empirical formula for propagation loss in land mobile radio services," IEEE Trans. Vech. Technol., vol. 29, pp. 317-325, August 1980.

[8] M D Springer, "The Algebra of Random Variables," Wiley, New York, 1979.

[9] A Sathyendran, K W Sowerby and M Shafi, "A Statistical Approach to the Analysis of DS/CDMA Cellular Systems Employing RAKE Receivers and Sectorised Antennas," Accepted for publication in IEEE Transactions on Vehicular Technology.

[10] A Papoulis, "Probability, Random Variables, and Stochastic Processes," third edition, McGraw-Hill, 1991. 